\title{
Seeing red in cyclic stratigraphy: Spectral noise estimation for astrochronology
}

\author{
Stephen R. Meyers ${ }^{1}$ \\ Received 27 February 2012; revised 15 June 2012; accepted 19 June 2012; published 30 August 2012.
}

[1] Fundamental to the development of astronomical time scales is the recognition of oscillatory variability within stratigraphic data and its evaluation relative to a null "noise" hypothesis. In this study, Monte Carlo simulations are used to investigate the suitability of two commonly used noise hypotheses (the "conventional" and "robust" AR1 approaches), and the results highlight important limitations in both for cyclostratigraphic application. Perhaps most problematic, the robust AR1 method can result in inflated confidence level estimates and excessive clumping of false positives within the low frequency portion of the spectrum, especially when the underlying noise process has a high lag-1 autocorrelation. Given typical cyclostratigraphic records, this technique will often impose "significant" eccentricity band variability, even in the case of pure AR1 noise. An alternative spectral noise estimation method is proposed to overcome these problems, which simultaneously allows for departures from the AR1 assumption, and obtains high statistical power-that is, the ability to accurately identify astronomical signals when they are present in the data. We apply the method to un-tuned $\delta^{18} \mathrm{O}$ data from Miocene sediments of the Ceara Rise, indicating statistically significant spectral power at frequencies that are consistent with the published orbital interpretation of Weedon et al. (1997). Furthermore, evaluation of the frequency arrangement of the significant spatial bedding periods, using the average spectral misfit method for astrochronologic testing, reveals that the null hypothesis of no orbital influence can be rejected with a high degree of confidence

(the $99.8 \%$ confidence level).

Citation: Meyers, S. R. (2012), Seeing red in cyclic stratigraphy: Spectral noise estimation for astrochronology,

Paleoceanography, 27, PA3228, doi:10.1029/2012PA002307.

\section{Introduction}

[2] Since the seminal work of Hays et al. [1976], spectral analysis has become one of the primary tools for hypothesis testing in cyclostratigraphy. A key step in cyclostratigraphic analysis and the development of astronomical time scales is the formulation of an appropriate null hypothesis against which to measure the statistical significance of power spectrum peaks. Numerous techniques are available (see Weedon [2003] for a review), but the most commonly utilized methods are based on autoregressive-1 (AR1) "red noise" models, mathematically defined as [Gilman et al., 1963; Mann and Lees, 1996]:

$$
\mathrm{r}_{\mathrm{n}}=\rho \mathrm{r}_{\mathrm{n}-1}+\omega_{n}
$$

\footnotetext{
${ }^{1}$ Department of Geoscience, University of Wisconsin-Madison, Madison, Wisconsin, USA.

Corresponding author: S. R. Meyers, Department of Geoscience, 1215 W. Dayton St., University of Wisconsin-Madison, Madison, WI 53706, USA. (smeyers@geology.wisc.edu)

C2012. American Geophysical Union. All Rights Reserved. 0883-8305/12/2012PA002307
}

where $\mathrm{n}=1, \ldots, \mathrm{N}$ is the time increment, $\mathrm{r}_{\mathrm{n}}$ is the red noise sequence, $0 \leq \rho<1$ is the lag- 1 autocorrelation coefficient, and $\omega_{\mathrm{n}}$ is a Gaussian white noise sequence.

[3] These models represent a simple stochastic process that is physically motivated by climate [Hasselmann, 1976] and depositional system dynamics [Thakur and Scheidegger, 1970; Sadler and Strauss, 1990], where a white noise source (e.g., weather) is integrated by an Earth System component with a slower response time (e.g., the oceans). The characteristic increase in power with decreasing frequency is what classifies AR1 processes as "red noise," and the power spectrum $\left(S_{A R 1}(f)\right)$ is defined as [Gilman et al., 1963; Bartlett, 1978; Mann and Lees, 1996]:

$$
S_{A R 1}(f)=S_{o} \frac{1-\rho^{2}}{1-2 \rho \cos \left(\pi f / f_{N}\right)+\rho^{2}}
$$

where $f$ denotes frequency, $f_{N}$ the Nyquist frequency (the highest frequency resolvable), and $S_{o}$ is the average power across the entire spectrum. Here, the $\rho$ parameter controls the shape of the spectrum, while $S_{o}$ defines the average vertical displacement from the abscissa (compare Figures 1b, 1e, and 1h). Both of these parameters must be estimated empirically 

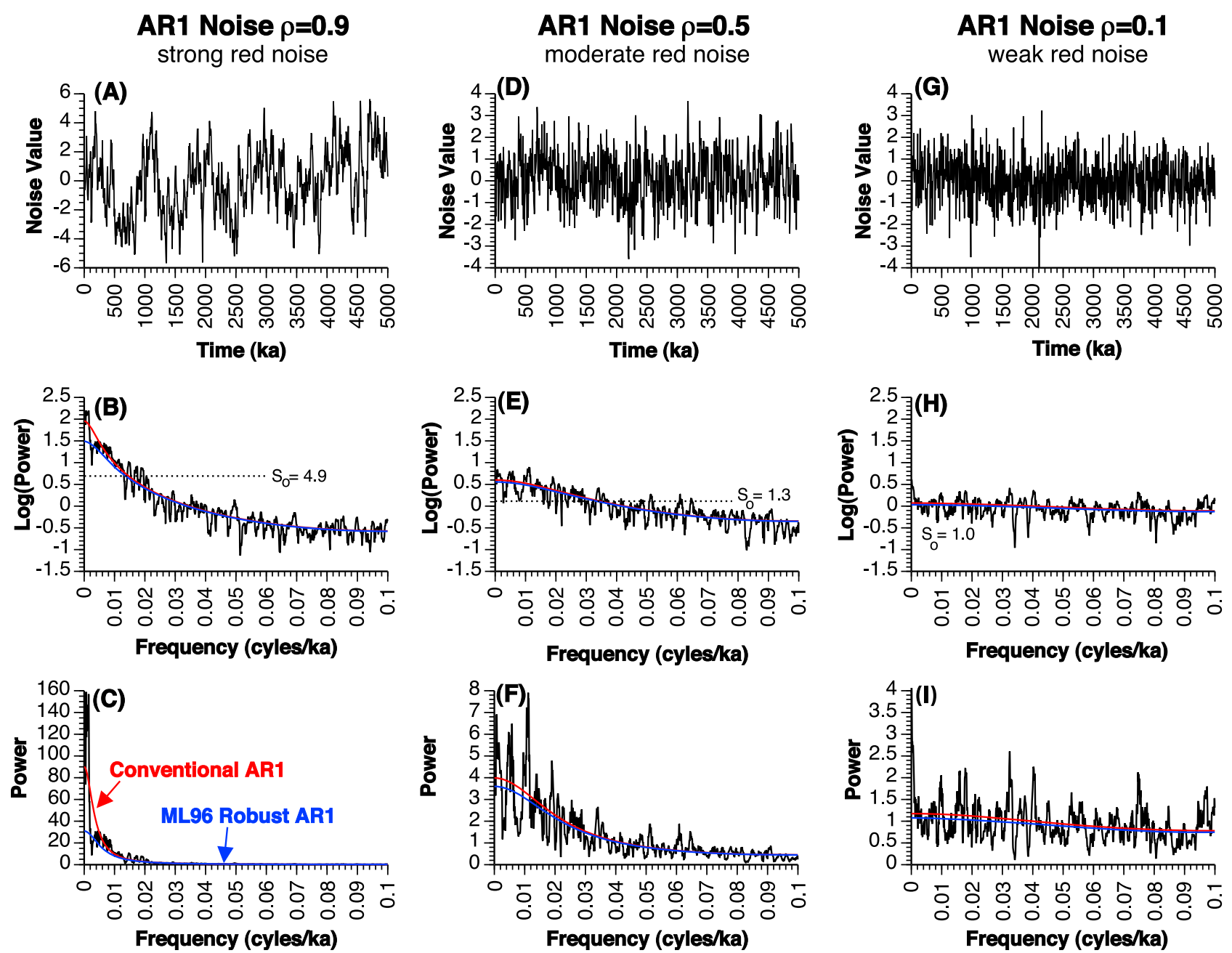

Figure 1. Example simulations of AR1 models with lag-1 autocorrelation coefficients $(\rho)$ of (a) 0.9 , (d) 0.5 and (g) 0.1. (b, e, h) The $3 \pi$ multitaper method (MTM) [Thomson, 1982] power spectra of the noise realizations, plotted as $\log$ (power) and (c, f, i) plotted as linear power. Red lines indicate the background as estimated using the conventional AR1 approach, and the blue lines indicate the background as estimated using the ML96 robust AR1 method. The three AR1 simulations have been assigned distinct $S_{0}$ values for the sake of illustration, but all other simulations in this study utilize AR1 noise with $S_{o}$ normalized unity, to facilitate comparison among the results.

from the data, and any bias imposed via the estimation procedure will necessarily result in bias in the reconstructed confidence levels.

[4] As demonstrated by Mann and Lees [1996, hereafter ML96], the conventional approach for estimation of $S_{A R 1}(f)$ can be substantially biased if the data series also contains a strong periodic or quasiperiodic signal, a circumstance that is presumed common in cyclostratigraphic records. As a solution, Mann and Lees [1996] propose a least squares analytic fit of equation (2) to a median-smoothed spectrum estimate, which deemphasizes strong peaks in the power spectrum (this follows the algorithm as updated by Ghil et al. [2002] and as implemented in the SSA-MTM Toolkit). The robust estimate of $\rho\left(\rho_{M L 96}\right)$ is the value that results in the smallest mean square error, and $S_{o-M L 96}$ is simply the mean value of the median smoothed power spectrum.
[5] While the conventional and ML96 robust AR1 models are utilized extensively in cyclostratigraphic research, few rigorous exploratory computational experiments have been conducted to evaluate their suitability (one notable exception is Vaughan et al. [2011]). Here we utilize Monte Carlo simulations to critically evaluate these two noise modeling approaches for use in astrochronology. As will be demonstrated, ensemble simulation results indicate that the robust method is generally preferable for estimation of $\rho$ and it has a greater ability to accurately identify orbital signals when they are in fact present in the data. However, the experiments also highlight some important limitations for cyclostratigraphic application, including inflated confidence level estimates (greater than expected false positive rate) and excessive clumping of false positives within the eccentricity band of the spectrum, especially given the strong red noise 
( $\rho$ of $0.7-0.9$ ) common in cyclostratigraphic records. To overcome these problems a new noise estimation method is adopted, based on a robust locally weighted regression estimate of the spectral background [Cleveland, 1979; Ruckstuhl et al., 2001].

\section{Monte Carlo Experiments: False Positives and Statistical Power}

\subsection{Simulation Setup}

[6] If confidence levels are to be meaningful, they must accurately reflect the chance occurrence of false positives (e.g., a 95\% confidence level should indicate that there is a $5 \%$ chance of erroneously rejecting the null hypothesis), and they should not artificially favor false positives in any particular region of the spectrum. An additional desirable attribute is high statistical power - that is, the ability of the metric to accurately identify orbital signals when they are in fact present in the data. In this study, false positive rate and spectral distribution are evaluated using 1000 Monte Carlo simulations of pure AR1 noise for $\rho$ of $0.9,0.5$ and 0.1 , representing strongly red, moderately red, and nearly white noise (we refer to these simulations as $\mathrm{AR} 1_{\mathrm{x}}$, with subscript $x$ indicating the $\rho$ value). Examples of these simulations are shown in Figure 1. To evaluate the influence of an imbedded astronomical signal on the estimated noise parameters ( $\rho$ and $S_{o}$ ), we examine a normalized eccentricity, tilt and precession signal (ETP) [Laskar et al., 2004; Meyers and Hinnov, 2010] combined with AR1 noise realizations, also with $\rho$ of $0.9,0.5$ and 0.1 (the ETP and AR1 signals are given equal variance before they are combined; we refer to these simulations as ETP-AR $1_{\mathrm{x}}$, with subscript $x$ indicating the $\rho$ value). Finally, to quantify statistical power we evaluate the ETP-AR $1_{0.9}$ model, as this is the most challenging case, and is characteristic of many cyclostratigraphic data sets.

[7] Simulated records are 5 million years long, with a temporal resolution of $5 \mathrm{ka}$, a representative sampling that is typical of many cyclostratigraphic investigations. The record length and number of simulations (1000) have been selected so as to allow resolution of all major astronomical cycles, to stabilize parameter estimates, and to provide small enough variance (uncertainties) for reliable method inter-comparison. In addition, assessment of statistical power is also conducted using 1 million yearlong ETP-AR $1_{0.9}$ simulations, a duration that is representative of many shorter cyclostratigraphic records. Summaries of the Monte Carlo experiments are provided in Figure 2 and Tables 1-3. Additional details on the ML96 analysis parameters, and noise simulation procedure utilized here, are included in Appendix A.

\subsection{Monte Carlo Simulation Results}

[8] As expected based on theory [Bartlett, 1978], the conventional AR1 approach provides unbiased estimates of $\rho$ and $S_{o}$ (here referred to as $\rho_{\text {raw }}$ and $S_{o-\text { raw }}$; Table 1 and Figure 2a) for the simulations of pure red noise, and therefore results in unbiased confidence level estimates (not shown). However, evaluation of the ETP-AR1 simulations demonstrates that both parameters become strongly biased by the presence of quasiperiodic variability across the spectrum. This bias is especially pronounced for the ETP$\mathrm{AR} 1_{0.9}$ simulations, where the median $\rho_{\text {raw }}$ is estimated as 0.75 , and the median $S_{\text {o-raw }}$ is approximately two times the true value (Table 1 and Figure 2b). The consequence is a reduction in statistical power (e.g., see the $1 \mathrm{Myr}$ and $5 \mathrm{Myr}$ simulations in Table 3), particularly at the eccentricity periods, and also the potential for enhanced false positives in portions of the spectrum due to the strongly biased estimate of the red noise spectrum shape $(\rho)$ [see, e.g., Mann and Lees, 1996]. In contrast, the ML96 robust red noise approach yields a median $\rho_{M L 96}$ value of 0.82 for the ETPAR $1_{0.9}$ simulations, closer to the true value of 0.9 , and the median $S_{O-M L 96}$ is reduced to 0.9 (compare to 1.97 for $S_{\text {o-raw }}$; Table 1). Both of these factors contribute to the enhanced statistical power of the ML96 method (Table 3). Note that such large $\rho$ estimates are typical of cyclostratigraphic records (e.g., see section 4).

[9] While this result would seem to favor the ML96 robust AR1 technique, another perspective is provided by applying the method to pure AR1 noise. For example, in the case of the $\mathrm{AR} 1_{0.9}$ simulations, the median $\rho_{M L 96}$ is also reduced to 0.82 , and the median $S_{O-M L 96}$ is underestimated by approximately half (Table 1 and Figures $2 \mathrm{a}$ and $2 \mathrm{~b}$ ). The consequence is a substantial enhancement of the false positive rate -approximately double that expected for the $90 \%$ and $95 \%$ confidence levels (Table 2 and Figure 2c) - and a clumping of false positives at the lowest frequencies (Figure 2d), the location of the short and long eccentricity terms in cyclostratigraphic spectra. Using this approach, one is essentially guaranteed to find statistically significant eccentricity band power in pure AR1 noise when the true $\rho$ is near 0.9 . The reason for this can be understood by evaluating Figure 1b, which illustrates the diminishment of low frequency power in the ML96 noise estimate due to the median smoothing methodology (see Appendix A and Mann and Lees [1996]). An enhanced false positive rate is also observed in the $\mathrm{AR} 1_{0.5}$ and $\mathrm{AR} 1_{0.1}$ simulations (Table 2 and Figure 2), and while the latter displays uniform spectral distribution of the false positives (Figure 21), the former is characterized by a slight enhancement at lower frequencies (Figure 2h).

[10] These results highlight important limitations in both conventional and robust red noise estimation methods. Clearly, if a pure AR1 process is present it is optimal to estimate the spectral background using the conventional AR1 approach, as the ML96 robust method is biased. However, if a mixed signal is present, the ML96 robust method provides a less biased estimate of the spectral background and enhanced statistical power. The essential problem is that we don't know, a priori, which of these two cases is correct. The ideal method should accurately reconstruct the background spectrum without such knowledge. This motivates the development of an alternative method for spectral background estimation based on robust locally weighted regression techniques [Cleveland, 1979], inspired by the original methods employed in Hays et al. [1976] and the robust estimation philosophy of Mann and Lees [1996].

\section{Robust Locally-Weighted Regression Spectral Background Estimation (LOWSPEC)}

[11] In their original analysis of late Pleistocene climate proxy data from the Southern Ocean, Hays et al. [1976] evaluated the significance of power spectrum peaks using a two-step approach. First, they pre-whitened the data set with a first difference filter to remove low frequency "red noise" 


\section{Ensemble \\ $\rho$ Estimates}
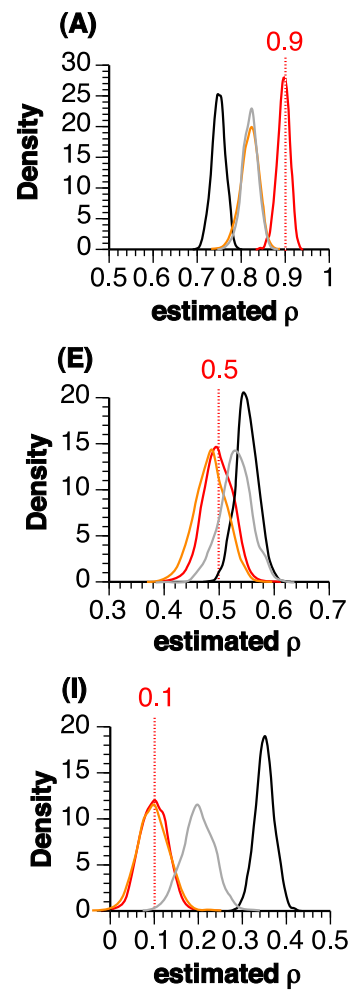

Ensemble $S_{0}$ Estimates
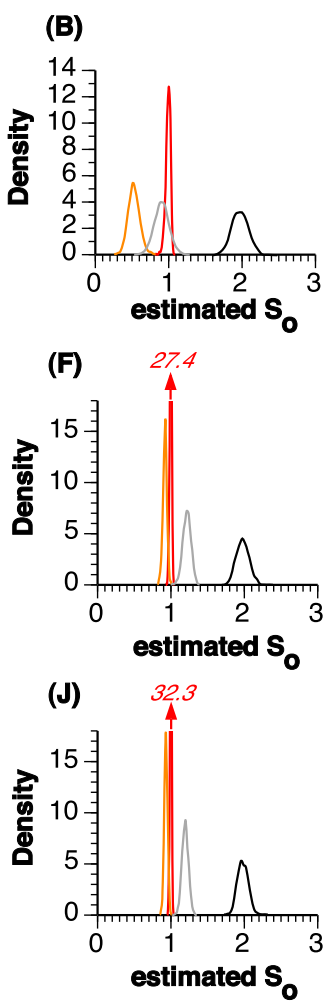
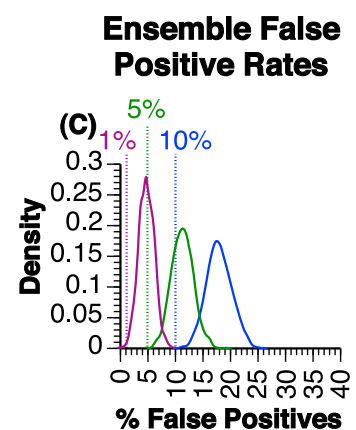

(G)

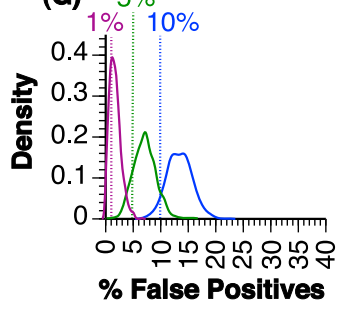

(K) $5 \%$

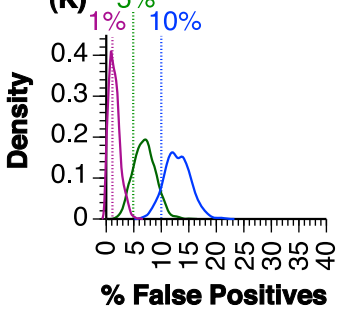

Ensemble False Positive Distribution
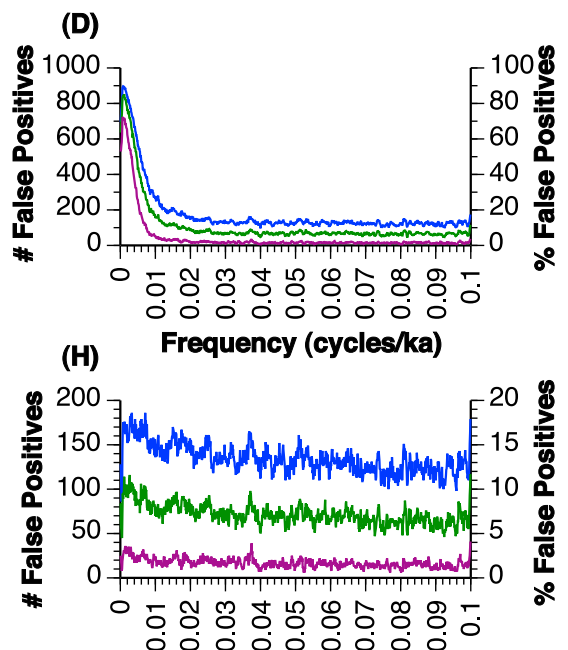

(L)

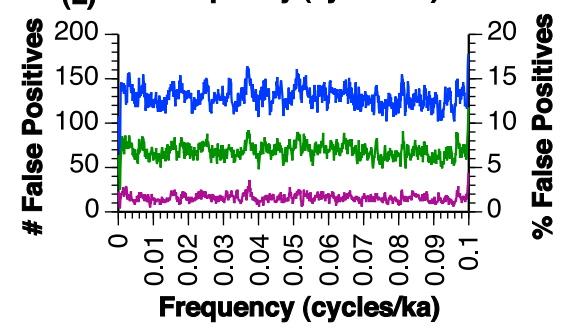

Frequency (cycles/ka)

\footnotetext{
- Conventional AR1 estimates: noise only

- ML96 AR1 estimates: noise only

- Conventional AR1 estimates: ETP + noise — ML96 AR1 estimates: ETP + noise
}

Figure 2. Simulation ensemble results for conventional AR1-based confidence level estimates and the ML96 robust AR1 confidence levels. (top) Summary of the AR $1_{0.9}$ and ETP-AR $1_{0.9}$ simulations, (middle) summary of the $\mathrm{AR} 1_{0.5}$ and ETP-AR $1_{0.5}$ simulations, and (bottom) summary of the AR $1_{0.1}$ and ETPAR $1_{0.1}$ simulations. (a) Kernel density estimates of $\rho$ for $A R 1_{0.9}$ and ETP-AR $1_{0.9}$ simulations. (b) Kernel density estimates of average power $\left(\mathrm{S}_{\mathrm{o}}\right)$ for $\mathrm{AR} 1_{0.9}$ and ETP-AR $1_{0.9}$ simulations. (c) Kernel density estimates of the false positive rate for the $99 \%, 95 \%$ and $90 \%$ confidence levels, using $\mathrm{AR} 1_{0.9}$. (d) Spectral distribution of false positives for the $99 \%, 95 \%$, and $90 \%$ confidence levels, using AR $1_{0.9}$. (e-1) The same format as Figures 2a-2d but for different $\rho$ values. As illustrated in Figures 2a, 2e, and 2i, the conventional $\rho_{\text {raw }}$ estimates determined from mixed ETP-AR1 signals can be biased either high or low. For a given record, the direction and magnitude of this bias will depend upon a range of factors, including the true $\rho$ value, the sampling rate, the specific timescale of each cyclic signal and its relative strength.

power that dominates the spectrum and strongly influences estimates of the spectral background. Application of a first difference filter is equivalent to removing an AR1 process with a $\rho$ of 1 (equation (1)). Following this pre-whitening, the residual background was estimated using a "low resolution" Blackman-Tukey spectrum [Blackman and Tukey, 1958]. The application of this spectrum smoothing approach to the pre-whitened data, rather than the explicit AR1 model fit that is now commonly used in cyclostratigraphy, has the advantage of allowing for departures from the AR1 model assumption [e.g., Vaughan et al., 2011] if it is not entirely valid. However, an important limitation of this approach is that the spectral background is estimated directly from a spectrum that includes a mixture of
Table 1. Median $\rho$ and $\mathrm{S}_{\mathrm{o}}$ Estimates for the Simulations, Using the Conventional AR1 and ML96 Robust AR1 Methods ${ }^{\mathrm{a}}$

\begin{tabular}{lccccc}
\hline \multirow{2}{*}{ Simulation } & \multicolumn{2}{c}{ Conventional AR1 } & & \multicolumn{2}{c}{ ML96 Robust AR1 } \\
\cline { 2 - 3 } \cline { 6 - 6 } & $\rho_{\text {raw }}$ & $\mathrm{S}_{\text {o-raw }}$ & & $\rho_{\text {ML96 }}$ & $\mathrm{S}_{\text {o-ML96 }}$ \\
\hline AR1 $_{0.9}$ & 0.90 & 1.00 & & 0.82 & 0.53 \\
ETP-AR1 $_{0.9}$ & 0.75 & 1.97 & & 0.82 & 0.90 \\
AR1 $_{0.5}$ & 0.50 & 1.00 & & 0.49 & 0.92 \\
ETP-AR1 $_{0.5}$ & 0.55 & 1.98 & & 0.53 & 1.21 \\
AR1 $_{0.1}$ & 0.10 & 1.00 & & 0.10 & 0.94 \\
ETP-AR1 & 0.35 & 1.98 & & 0.20 & 1.19 \\
\hline
\end{tabular}

${ }^{a}$ All simulations are evaluated using $3 \pi$ MTM spectra. 
Table 2. Median False Positive Rates for the Simulations, Using the ML96 Robust AR1 and LOWSPEC Methods ${ }^{\mathrm{a}}$

\begin{tabular}{cccccccc}
\hline & \multicolumn{3}{c}{ ML96 Robust AR1 } & & \multicolumn{3}{c}{ LOWSPEC } \\
\cline { 2 - 4 } \cline { 6 - 8 } Simulation & $90 \%$ CL & $95 \%$ CL & $99 \%$ CL & & $90 \%$ CL & $95 \%$ CL & $99 \%$ CL \\
\hline AR1 $1_{0.9}$ & $17.8 \%$ & $11.4 \%$ & $4.7 \%$ & & $10.3 \%$ & $5.1 \%$ & $0.9 \%$ \\
AR1 $_{0.5}$ & $13.4 \%$ & $7.2 \%$ & $1.6 \%$ & & $10.4 \%$ & $5.1 \%$ & $0.9 \%$ \\
AR1 $_{0.1}$ & $12.8 \%$ & $6.8 \%$ & $1.4 \%$ & & $10.4 \%$ & $5.1 \%$ & $0.9 \%$ \\
\hline
\end{tabular}

${ }^{\mathrm{a}}$ All simulations are evaluated using $3 \pi$ MTM spectra.

background and hypothesized quasiperiodic variability, without allowance for sharp spectral features that should be excluded from the background estimation. Thus, the statistical power of the technique is necessarily reduced [Weedon, 2003].

[12] The statistical power can be enhanced if a robust methodology is used in the estimation of the spectral background [Box, 1953; Mann and Lees, 1996], that is, a technique that can identify and exclude extreme departures from the spectral background during the smoothing process. A suitable smoothing approach is provided by robust locally weighted regression [Cleveland, 1979; Ruckstuhl et al., 2001], defined as:

$S_{\text {background }}\left(f_{o}\right)=\underset{\beta}{\operatorname{argmin}} \sum_{i=1}^{n} w\left(f_{i}-f_{o}\right)\left\{S\left(f_{i}\right)-\left[\beta_{0}+\left(\beta_{1}\left(f_{i}-f_{o}\right)\right]\right\}^{2}\right.$

where $n$ is the number of frequencies evaluated for each local regression fit, $w\left(f_{i}-f_{o}\right)$ is a weighting function for each frequency $\left(f_{i}\right)$ included in the background fit at frequency $f_{o}$, and $\beta$ are the 'local' regression coefficients. Importantly, the weights $w\left(f_{i}-f_{o}\right)$ are determined iteratively and incorporate a robustness criterion to identify and exclude extreme departures, for example, due to narrowband spectral features that are attributable to periodic or quasiperiodic variability. A detailed introduction to the method is provided in Cleveland [1979], and a relevant example of its application for spectral background estimation in quantitative spectroscopy can be found in Ruckstuhl et al. [2001].

[13] For the algorithm adopted here, pre-whitening is conducted using the conventional $\rho$ estimator, and departures from this AR1 model are subsequently compensated for using robust locally weighted regression (equation (3)). This approach is essentially similar to the "combined parametric/ non-parametric" method advocated by Percival and Walden [1993, section 9.10] [see also Thomson et al., 2001]. Obviously, the use of AR1 pre-whitening is most appropriate when testing against an AR1 model, but other types of prewhitening can be utilized for the evaluation of a wide range of noise models [Percival and Walden, 1993]. Additional details on the algorithm are contained in Appendix B.

[14] Results of 1000 Monte Carlo simulations of $A R 1_{0.9}$ noise indicate median false positive rates of $0.9 \%, 5.1 \%$ and $10.3 \%$ for the LOWSPEC $1 \%, 5 \%$ and $10 \%$ confidence levels (Figure 3a and Table 2), substantially lower than those of the ML96 robust method. Furthermore, the distribution of false positives is uniform across the spectrum (Figure $3 b$ ). Finally, evaluation of the 1 Myr ETP-AR $1_{0.9}$ simulations indicates that the LOWSPEC statistical power for detection of the long eccentricity term exceeds that of the ML96 method, while detection of the short eccentricity terms is somewhat diminished, and all methods demonstrate exceptional statistical power with regards to the dominant obliquity ('o2') and precession terms (Table 3). However, while the high frequency orbital terms are most consistently identified in the ensemble simulations, it is important to note that sedimentation rate instability in real stratigraphic data will most strongly affect and smear these high frequency terms, a necessary consequence of which is a reduction of statistical power.

[15] The longer $5 \mathrm{Myr}$ simulations in general demonstrate high statistical power for all methods, which can be understood as due to an increase in the number of observed cycles at all orbital periods, and the consequent sharpening of their

Table 3. Statistical Power of the Conventional AR1, ML96 Robust AR1 and LOWSPEC Methods Relative to the 95\% and 99\% Confidence Level $^{\mathrm{a}}$

\begin{tabular}{|c|c|c|c|c|c|c|c|c|c|c|c|c|c|}
\hline \multirow[b]{2}{*}{ Term } & \multirow{2}{*}{$\begin{array}{l}\text { Period }^{\mathrm{b}} \\
\text { (ka) }\end{array}$} & \multicolumn{3}{|c|}{$\begin{array}{c}1 \text { Million Year Simulations: } \\
\text { 95\% CL }\end{array}$} & \multicolumn{3}{|c|}{$\begin{array}{c}1 \text { Million Year Simulations: } \\
\text { 99\% CL }\end{array}$} & \multicolumn{3}{|c|}{$\begin{array}{c}5 \text { Million Year Simulations: } \\
\text { 95\% CL }\end{array}$} & \multicolumn{3}{|c|}{$\begin{array}{c}5 \text { Million Year Simulations: } \\
\text { 99\% CL }\end{array}$} \\
\hline & & AR1 & ML96 & LOWSPEC & AR1 & ML96 & LOWSPEC & AR1 & ML96 & LOWSPEC & AR1 & ML96 & LOWSPEC \\
\hline e1 & 404 & 0.137 & 0.586 & 0.749 & 0.002 & 0.387 & 0.484 & 0.748 & 0.953 & 0.993 & 0.428 & 0.860 & 0.942 \\
\hline e2 & 125 & $0.040^{\mathrm{c}}$ & $0.592^{\mathrm{c}}$ & $0.481^{\mathrm{c}}$ & $0.001^{\mathrm{c}}$ & $0.231^{\mathrm{c}}$ & $0.155^{\mathrm{c}}$ & 0.069 & 0.887 & 0.762 & 0.011 & 0.657 & 0.441 \\
\hline e3 & 95 & $0.040^{\mathrm{c}}$ & $0.592^{\mathrm{c}}$ & $0.481^{\mathrm{c}}$ & $0.001^{\mathrm{c}}$ & $0.231^{\mathrm{c}}$ & $0.155^{\mathrm{c}}$ & 0.605 & 1.000 & 0.996 & 0.210 & 0.994 & 0.952 \\
\hline o1 & 54 & 0.000 & 0.021 & 0.005 & 0.000 & 0.005 & 0.002 & 0.011 & 0.958 & 0.714 & 0.000 & 0.819 & 0.357 \\
\hline o2 & 41 & 1.000 & 1.000 & 1.000 & 1.000 & 1.000 & 1.000 & 1.000 & 1.000 & 1.000 & 1.000 & 1.000 & 1.000 \\
\hline p1 & 24 & $1.000^{\mathrm{c}}$ & $1.000^{\mathrm{c}}$ & $1.000^{\mathrm{c}}$ & $1.000^{\mathrm{c}}$ & $1.000^{\mathrm{c}}$ & $1.000^{\mathrm{c}}$ & 1.000 & 1.000 & 1.000 & 1.000 & 1.000 & 1.000 \\
\hline $\mathrm{p} 2$ & 22 & $1.000^{\mathrm{c}}$ & $1.000^{\mathrm{c}}$ & $1.000^{\mathrm{c}}$ & $1.000^{\mathrm{c}}$ & $1.000^{\mathrm{c}}$ & $1.000^{\mathrm{c}}$ & 1.000 & 1.000 & 1.000 & 1.000 & 1.000 & 1.000 \\
\hline p3 & 19 & 1.000 & 1.000 & 1.000 & 0.999 & 1.000 & 1.000 & 1.000 & 1.000 & 1.000 & 1.000 & 1.000 & 1.000 \\
\hline
\end{tabular}

\footnotetext{
${ }^{\mathrm{a}} \mathrm{An}$ orbital signal is considered detected if significant power is achieved within a bandwidth defined by the half power points of the theoretical astronomical signal [Laskar et al., 2004]. A value of 1 indicates perfect detection. The 1 Myr simulations use $2 \pi$ MTM spectra, and the 5 Myr simulations use $3 \pi$ MTM spectra. For the $\mathrm{p} 1$ and $\mathrm{p} 2$ terms in the $1 \mathrm{Myr}$ simulations, a bandwidth larger than the half power points is used to provide a more suitable statistical power test. This is necessitated by the overlap of the $\mathrm{p} 1$ and $\mathrm{p} 2$ terms within the spectral bandwidth which creates a prominent superposed peak in the region of overlap. While the e2 and e3 terms in the 1 Myr simulations also exhibit this phenomena, the half power points are sufficient to capture most of the orbital signal bandwidth.

${ }^{b}$ Based on $3 \pi$ MTM analysis of the Laskar et al. [2004] solution, 0-5 Ma.

${ }^{\mathrm{c}}$ The e 2 and e 3 terms are not individually distinguishable in the power spectrum, given the spectral bandwidth resolution of the $1 \mathrm{Myr}$ simulations. The same is true for the $\mathrm{p} 1$ and $\mathrm{p} 2$ terms.
} 


\section{(A) Ensemble False}

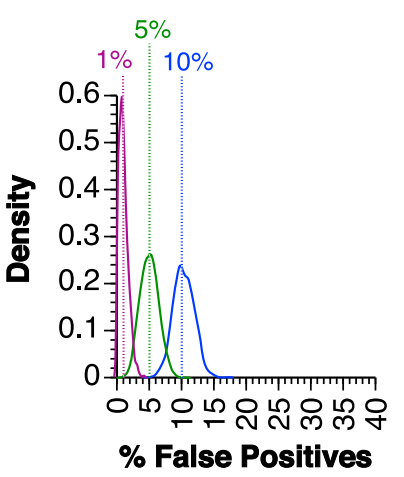

(B) Ensemble False

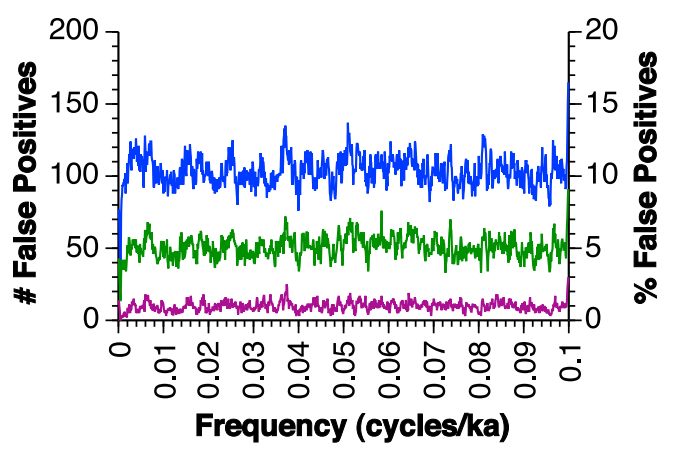

\footnotetext{
- False positives using LOWSPEC 99\% Confidence Leve

— False positives using LOWSPEC 95\% Confidence Level

- False positives using LOWSPEC 90\% Confidence Level
}

Figure 3. $\mathrm{AR}_{0.9}$ simulation ensemble results for LOWSPEC-based confidence level estimates. (a) Kernel density estimates of the false positive rate for the $99 \%, 95 \%$ and $90 \%$ confidence levels. (b) Spectral distribution of false positives for the $99 \%, 95 \%$, and $90 \%$ confidence levels.

associated spectral features. However, for these $5 \mathrm{Myr}$ simulations the conventional AR1 method indicates a degree of degradation in the statistical power associated with detection of the eccentricity periods, especially the ' $\mathrm{e} 2$ ' term (Table 3). In contrast, all of the eccentricity terms are clearly detected using the ML96 and LOWSPEC approaches (Table 3).

\section{Testing for an Astronomical Signal in Ceara Rise $\delta^{18} O$ Data}

\subsection{Evaluation Against the Red Noise Hypothesis}

[16] As a test case for the LOWSPEC method we revisit early Miocene proxy data from the equatorial Atlantic Ceara Rise, ODP Site 926B [Pälike et al., 2006], a site that has been instrumental in the development of astrochronologies for the Miocene and Oligocene time scale [Weedon et al., 1997; Shackleton et al., 1999], and has also provided constraints on the theoretical astronomical solutions [Laskar et al., 1993, 2004]. The original cyclostratigraphic work at this site was conducted by Weedon et al. [1997], which identified a statistically significant bedding period of $\sim 1 \mathrm{~m}$ (relative to a conventional AR1 model) in depth-scaled reflectance data. This cycle was interpreted as due to obliquity forcing based on biostratigraphically estimated sedimentation rates [Curry et al., 1995]. Subsequent studies by Shackleton et al. [1999] and Pälike et al. [2006] further developed the astrochronology at Ceara Rise by tuning extended proxy records to theoretical orbital-insolation models [Laskar et al., 1993, 2004]. These astronomically tuned data have been used to support the accuracy of the theoretical orbital-insolation solutions, through the identification of a 2:1 orbital resonance between eccentricity $(2.4 \mathrm{Myr})$ and obliquity $(1.2 \mathrm{Myr})$ that is predicted by the astronomical models [Pälike et al., 2006]. However, it is important to note that validation of the Laskar et al. [2004, hereafter LA04] model using data that is tuned directly to it raises the potential for circularity. This motivates an independent assessment of the orbital hypothesis that is free of orbital tuning to LA04, as is provided here and in Meyers and Hinnov [2011].

[17] Evaluation of the Ceara Rise $\delta^{18} \mathrm{O}$ record (a proxy for ice volume and deep ocean temperature) [Pälike et al., 2006] using the conventional AR1 and ML96 robust AR1 methods is displayed in Figure 4. The two confidence level estimates (Figure 4d) are in close agreement for most of the spectrum, but substantially diverge at frequencies $<0.4$ cycles/meters. The ML96 confidence level estimates are elevated in the lower $10 \%$ of the spectrum, precisely the response we'd expect to observe if due to an enhanced false positive rate from strong red noise (compare with Figure 2d). The raw and ML96 robust $\rho$ coefficients for this record are 0.79 and 0.68 , respectively, and while both can be biased by the hypothesized orbital signal (see Figure 2a), they support the inference of relatively strong red noise. Important for the discussion that follows, the highly significant $\sim 0.8 \mathrm{~m}$ cycle in $\delta^{18} \mathrm{O}$ (peak "c" in Figure $4 \mathrm{~b}$ ) is consistent with a reflectance cycle that was previously hypothesized to represent the $41 \mathrm{ka}$ obliquity signal [Weedon et al., 1997]. If this is the case, then the strong power at $<0.4$ cycles $/ \mathrm{m}$ is in the eccentricity band (as well as the $\sim 1200 \mathrm{ka}$ obliquity "modulator" band; peaks "a" and "b" in Figure 4b), and thus it is critical to determine if this power is actually significant, or artificially inflated by the ML96 technique.

[18] Results of the LOWSPEC analysis are displayed in Figure 5. Following the pre-whitening procedure, the $\delta^{18} \mathrm{O}$ spectrum is slightly blue (displaying an increase in power with frequency). This result indicates that the lag-1 autocorrelation $(\rho)$ used for pre-whitening is slightly overestimated, and suggests that the robust $\rho$ estimate is more appropriate. One could iteratively modify the pre-whitening $\rho$ estimate until the spectrum is most closely white, although this approach is not pursued here. Instead, the slight overestimation of $\rho$ is compensated for by the locally weighted 

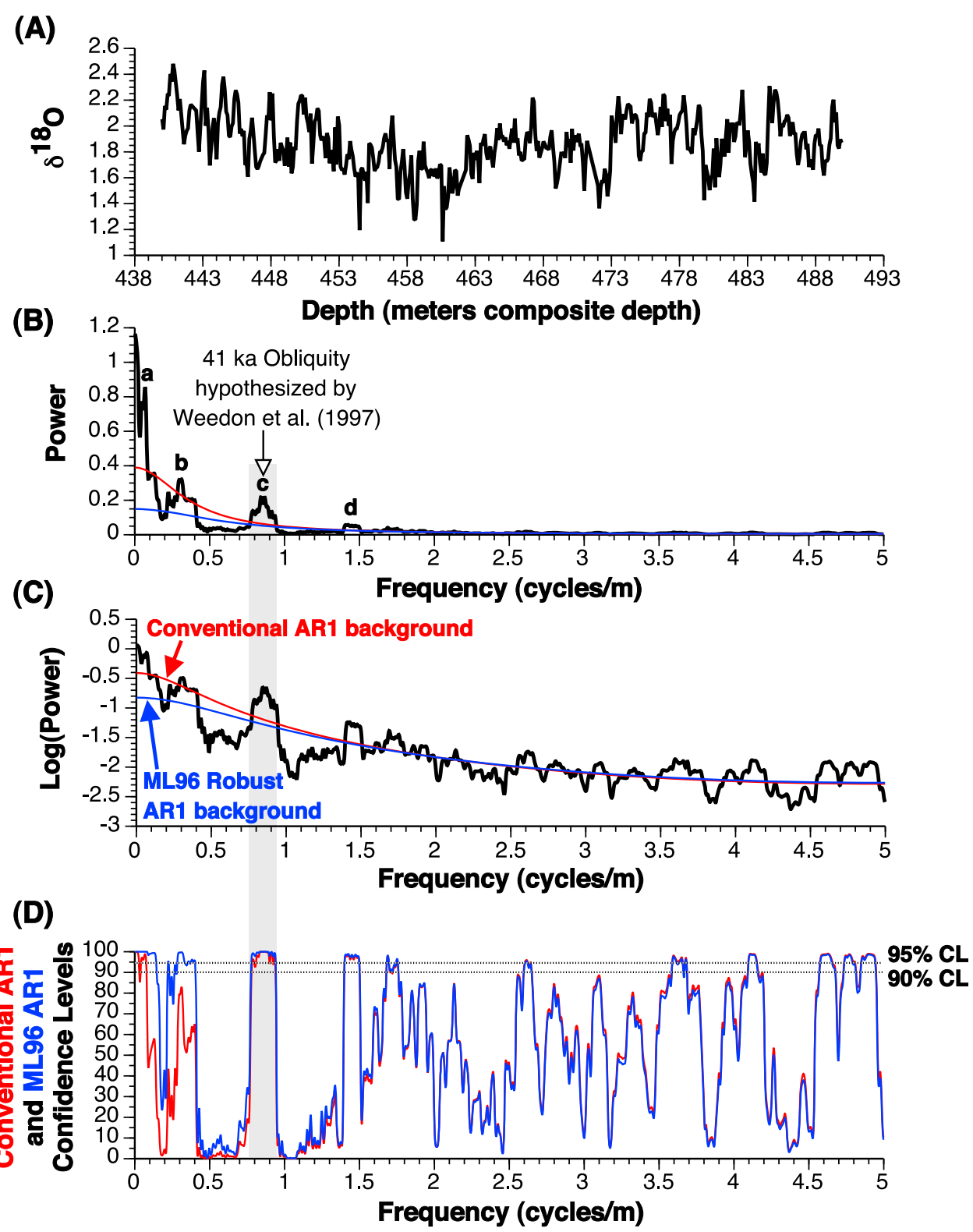

Figure 4. Conventional AR1 and ML96 robust AR1 confidence level estimates for Ceara Rise benthic foraminifera $\delta^{18} \mathrm{O}$ data (440-490 m composite depth). (a) The $\delta^{18} \mathrm{O}$ data (adjusted for seawater disequilibrium) versus meters composite depth [Pälike et al., 2006]. (b) The $3 \pi$ MTM power spectrum of the depth-scaled $\delta^{18} \mathrm{O}$ data, with conventional AR1 background (red) and ML96 robust AR1 background (blue). (c) Logarithmic plot of $3 \pi$ MTM power, with conventional AR1 background (red) and ML96 robust AR1 background (blue). (d) Confidence level estimates based on the conventional AR1 (red) and ML96 robust AR1 (blue) methods.

background estimation procedure (green lines in Figures 5a and $5 b)$.

[19] Comparison of the LOWSPEC confidence levels to the conventional AR1 and ML96 robust AR1 results indicates that all methods document highly significant $(>95 \% \mathrm{CL})$ peaks at $\sim 0.8$ cycles $/ \mathrm{m}$ ("c" in Figure $5 \mathrm{~b}$ ), $\sim 1.4$ cycles $/ \mathrm{m}$ ("d" in Figure $5 \mathrm{~b}$ ), $\sim 1.7$ cycles $/ \mathrm{m}$, and $<0.1$ cycles $/ \mathrm{m}$ ("a" in Figure $5 \mathrm{~b}$ ). The significance of the LOWSPEC $\sim 0.3 \mathrm{cycle} / \mathrm{m}$ term (peak "b" in Figures 4 and 5) is intermediate between that of the conventional $(83.0 \% \mathrm{CL})$ and ML96 robust AR1 (99.7\% CL) methods, achieving a confidence level of $95.1 \%$. Furthermore, it is important to note that this result is not affected by an enhanced false positive rate, as is the ML96 method (Figure 2 and Table 2). Finally, all frequencies above 3 cycles $/ \mathrm{m}$ display lower confidence levels in the LOWSPEC spectrum than those associated with the conventional AR1 or ML96 robust AR1 techniques (compare Figures $4 \mathrm{~d}$ and 5c). Importantly, these frequencies are outside of the proposed orbital band [Weedon et al., 1997].

\subsection{Testing the Astronomical Hypothesis at Ceara Rise}

[20] In interpreting spectral results such as those shown in Figure 5, it is necessary to define a confidence level threshold for the identification of plausible spatial bedding 

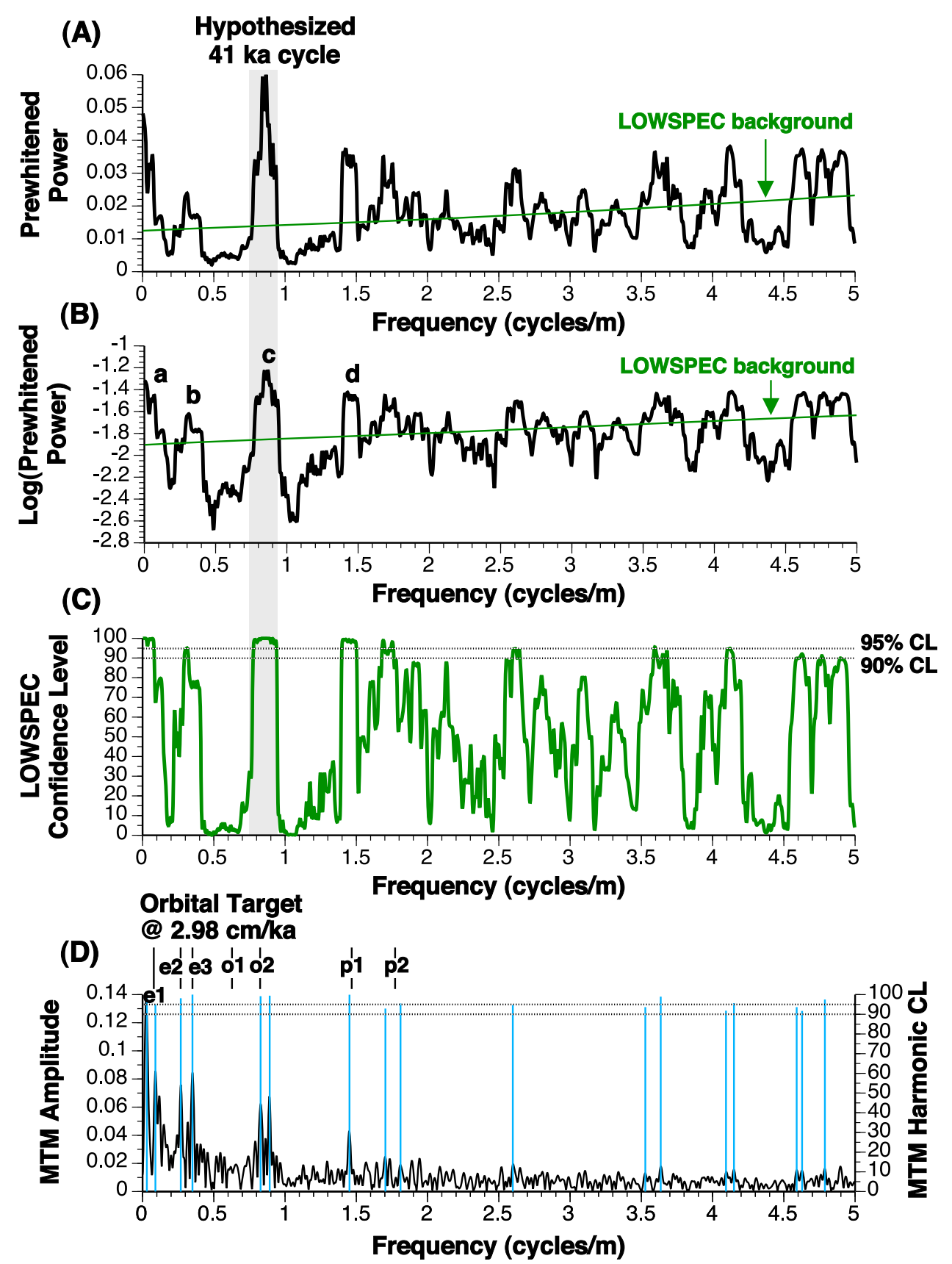

Figure 5. LOWSPEC confidence level estimates for Ceara Rise benthic foraminifera $\delta^{18} \mathrm{O}$ data (440-490 m composite depth). (a) The $3 \pi$ MTM power spectrum of the depth-scaled $\delta^{18} \mathrm{O}$ data following pre-whitening, with LOWSPEC background (green). (b) Logarithmic plot of $3 \pi$ MTM power following pre-whitening, with LOWSPEC background (green). (c) Confidence level estimates based on the LOWSPEC background estimate. (d) $3 \pi$ MTM harmonic analysis amplitude results (black), with MTM harmonic confidence levels (light blue) based on the harmonic $F$ test.

frequencies that may represent astronomical forcing or pacing. In cyclostratigraphic studies it is common to use the 90\% confidence level as this threshold [e.g., Hays et al., 1976; Park and Herbert, 1987; Thomson, 1990; Hinnov and Goldhammer, 1991; Sageman et al., 1997; Weedon and Jenkyns, 1999]. Whether this confidence level-or any particular level-represents an appropriate statistical criterion is debatable. For example, Thomson [1990, 2010],
Muller and MacDonald [2000], Mudelsee [2010], and Vaughan et al. [2011] highlight the important point that many independent statistical tests (potentially hundreds to thousands) are conducted during a single spectral analysis, and an allowance should be made for this by using a 'multiple test' criterion. That is, given 100 independent frequencies in a spectrum, one would expect at least 10 to exceed the $90 \%$ confidence level in the case of pure noise, 
Table 4. Average Spectral Misfit Results for the Ceara Rise $\delta^{18}$ O Data (440-490 m Composite Depth) ${ }^{\mathrm{a}}$

\begin{tabular}{|c|c|c|c|c|}
\hline $\begin{array}{l}\text { Frequency } \\
\text { (cycles/meter) }\end{array}$ & $\begin{array}{c}\text { MTM Harmonic } \\
\text { Probability }\end{array}$ & $\begin{array}{l}\text { Periodicity (ka) } \\
\mathrm{s}=2.98 \mathrm{~cm} / \mathrm{ka}^{\mathrm{b}}\end{array}$ & Orbital Target $^{\mathrm{c}}(\mathrm{ka})$ & Spectral Misfit $\alpha_{\mathrm{k}}($ cycles $/ \mathrm{ka})$ \\
\hline 0.030 & $98.16 \%$ & $1118.57(837.80-1682.37)$ & & \\
\hline 0.090 & $95.11 \%$ & $372.86(335.39-419.74)$ & $\mathrm{e} 1=404.18$ & 0 \\
\hline 0.268 & $98.13 \%$ & $125.21(120.69-130.09)$ & $\mathrm{e} 2=123.82$ & 0 \\
\hline 0.350 & $99.98 \%$ & $95.88(93.20-98.71)$ & $e 3=94.78$ & 0 \\
\hline 0.828 & $99.18 \%$ & $40.53(40.04-41.03)$ & $\begin{array}{l}\mathrm{o} 1=53.16 \\
\mathrm{o} 2=40.52\end{array}$ & $\begin{array}{c}5.865 \mathrm{E}-03 \\
0\end{array}$ \\
\hline 0.892 & $99.44 \%$ & $37.62(37.20-38.05)$ & & \\
\hline 1.452 & $99.93 \%$ & $23.11(22.95-23.27)$ & $\mathrm{p} 1=22.82$ & $5.43 \mathrm{E}-04$ \\
\hline 1.704 & $92.90 \%$ & $19.69(19.58-19.81)$ & & \\
\hline \multirow[t]{2}{*}{1.810} & $95.47 \%$ & $18.54(18.44-18.64)$ & $\mathrm{p} 2=18.91$ & $1.062 \mathrm{E}-03$ \\
\hline & & & & $\begin{array}{r}\Sigma \alpha_{\mathrm{k}}=7.470 \mathrm{E}-03 \\
\mathrm{n}=7 \\
\text { Average Spectral Misfit }=1.067 \mathrm{E}-03 \\
\mathrm{p}-\mathrm{value}=0.00200 \\
\text { Hypothesis Significance Level }=0.200\end{array}$ \\
\hline
\end{tabular}

${ }^{a}$ ASM analysis was conducted using the 17 frequencies identified in Figure 5d. Monte Carlo spectra simulations utilize 17 frequencies, randomly distributed, with the same resolution limitations as the Ceara Rise $\delta^{18} \mathrm{O}$ data. The significance levels are based on comparison of the observed ASM value with the probability distribution of ASM values from 100,000 simulated spectra (Figure 6). The orbital target (predicted orbital periods) for $22 \mathrm{Myr}$ is based on Berger et al. [1992], to provide an assessment that is independent of Laskar et al. [2004].

${ }^{b}$ Values in parentheses reflect the uncertainty of each estimated period (see Meyers and Sageman [2007] for further details).

${ }^{\mathrm{c}}$ Orbital target for $22 \mathrm{Ma}$ [Berger et al., 1992].

and thus one could employ a more stringent 'multiple test' $90 \%$ confidence level that accounts for this [e.g., Vaughan et al., 2011].

[21] In counterpoint, the 'multiple test' confidence levels assigned to these statistical hypotheses are only exact for time series - that is, records for which the time scale is perfectly known. When depth is used as a proxy for time, the assigned confidence levels do not strictly hold. Even a small amount of "jitter" imposed by sedimentation rate instability serves to smear and distort the preserved astronomical signals [Moore and Thomson, 1991; Meyers et al., 2001; Huybers and Wunsch, 2004], making their identification with a $90 \%$ 'multiple test' criterion challenging or impossible. Given typical sedimentation rate instability associated with pelagic and hemipelagic records, achievement of the 'standard' $90 \%$ confidence level may be the best we can hope for in many real stratigraphic data sets [e.g., Thomson, 1990, p. 571].

[22] For yet another perspective however, consider the following: even when depth-scaled data express a cycle that passes a selected 'multiple-test' criterion ( $90 \%, 95 \%$, etc.), such tests do not provide an evaluation of the temporal duration of the spatial cycle. The temporal period must be assumed or evaluated based on other geochronologic information, which is typically poorly constrained in deep-time studies. In other words, a statistical test that does not evaluate temporal hypotheses for the observed rhythms cannot permit an explicit test of the astronomical hypothesis.

[23] Given these issues, a practical and rigorous test for the presence of an astronomical signal must appeal to other aspects of the preserved rhythms, such as the expected hierarchy of cycles that the astronomical model predicts [Meyers and Sageman, 2007; Meyers, 2008; Malinverno et al., 2010]. In fact, assessment of cycle hierarchy was a key component of the original evaluation of late Pleistocene records by Hays et al. [1976], but only recently has a formal statistical framework for such hypothesis testing been proposed (average spectral misfit or ASM [Meyers and Sageman, 2007]; for a Bayesian approach see Malinverno et al. [2010]). Application of such methods recognizes an important distinction between testing the astronomical hypothesis, and prospecting for unknown 'cycles'-those which are not predicted based on a physical model. In the case of the former, there are additional features of the astronomical signal (beyond peak significance) that can be utilized in hypothesis testing [Hinnov, 2000].

[24] The ASM method for astrochronologic testing is used to evaluate the Ceara Rise $\delta^{18} \mathrm{O}$ data in this study, and we provide a very brief review of it here (see Meyers and Sageman [2007] for a detailed discussion). Following the ASM approach, observed spatial bedding periods are considered candidate astronomical cycles, which may or may not represent orbital influence. The discrepancy between the predicted orbital frequencies and the observed periods in the spectrum (termed 'average spectral misfit', in cycles/ka [Meyers and Sageman, 2007, equation 1]) is determined for a given temporal calibration, or a range of plausible calibrations. Finally, the observed ASM is compared to the ASM of Monte Carlo simulated spectra with the same number of significant frequencies as the observed spectrum, and identical resolution limitations, but with spectral peaks distributed at random frequencies. Importantly, any false positives that are recorded in the observed spectrum are also explicitly incorporated in the Monte Carlo simulation. The ensemble of Monte Carlo simulations then indicate how frequently a particular ASM value should occur by chance, given a spectrum with similar resolution and randomly distributed frequencies, thus providing a formal null hypothesis test of orbital influence.

[25] Selection of a specific noise confidence level (e.g., $90 \%, 95 \%$ ) for identification of candidate astronomical frequencies serves to influence the number of false positives included in the ASM analyses. Most importantly, the noise hypothesis test must not artificially impose a structure on the spectrum, as has been demonstrated for the ML96 robust AR1 method (Figure 2d) [see also Vaughan et al., 2011]. For the Ceara Rise $\delta^{18} \mathrm{O}$ data we use a multistep screening 


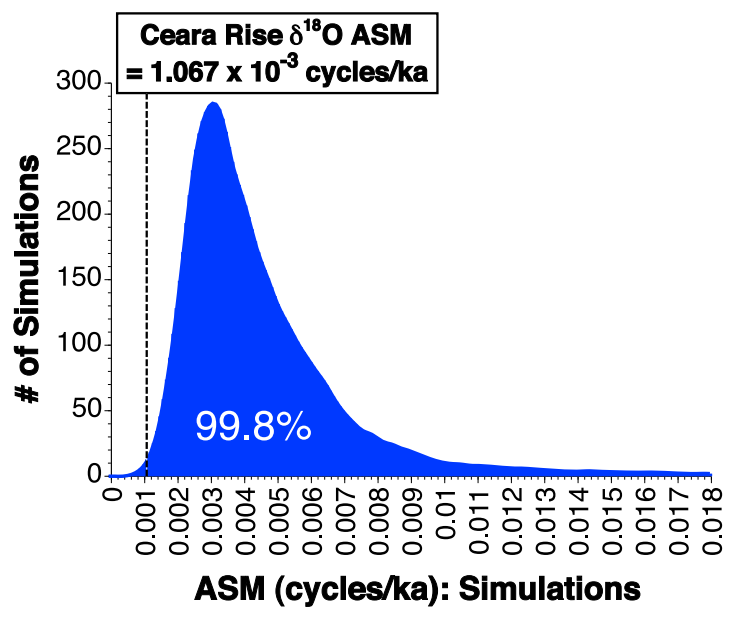

Figure 6. Probability distribution of the ASM results from 100,000 Monte Carlo simulated spectra. The observed ASM from the Ceara Rise $\delta^{18} \mathrm{O}$ data (440-490 m composite depth) is indicated for comparison; $99.8 \%$ of the simulations are characterized by an ASM value that exceeds the observed ASM.

approach to identify candidate frequencies, which greatly reduces false positives while also sensitive to the inherent limitations of cyclostratigraphic data. Specifically, we start by isolating those frequencies that achieve a $90 \%$ LOWSPEC confidence level, and also a 90\% MTM harmonic $F$ test confidence level. The MTM harmonic $F$ test is designed to test for phase-coherent sinusoids in white or colored noise [Thomson, 1982; Percival and Walden, 1993] (Figure 5d), and is, to a first order, independent of the LOWSPEC-based hypothesis test. The combination of these two distinct noise tests is an important advantage of the MTM approach for cyclostratigraphic analysis.

[26] In identifying candidate cycles for astrochronologic testing, we also make allowance for the smoothing inherent in the MTM power spectral estimate (Figure $5 b$ ), as compared to the MTM harmonic spectrum (Figure 5d). That is, an $F$ test peak is included in the ASM analysis if it achieves the $90 \%$ MTM harmonic confidence level, while also achieving the 90\% LOWSPEC confidence level within + /half the power spectrum bandwidth resolution. We include one additional criterion to further reduce the false positive rate, a requirement that significant $F$ tests must occur on a local power spectrum 'high', which we parameterize as occurring above the local LOWSPEC background estimate.

[27] Seventeen candidate frequencies meet the required criteria outlined above. If the orbital interpretation of Weedon et al. [1997] is correct, a sedimentation rate of $\sim 2.98 \mathrm{~cm} / \mathrm{ka}$ is appropriate for these strata, and this temporal calibration yields close visual agreement between observed frequencies and the proposed orbital target (Figure 5d). But what is the statistical significance of this observed fit? Table 4 summarizes the results of the average spectral misfit analysis, indicating an ASM value of $1.067 \times 10^{-3}$ cycles $/ \mathrm{ka}$; the largest discrepancy between the orbital target and the observed $\delta^{18} \mathrm{O}$ spectrum is for the longer obliquity period ('o1'), which is not surprising given the low statistical power of all methods in identifying this weak orbital term (e.g., see the 1 Myr simulations in Table 3). Finally, Monte Carlo simulation reveals that the likelihood of generating such a small
ASM value from a randomly organized spectrum is very small (Table 4 and Figure 6); only 200 of 100,000 simulated spectra achieve such low ASM values. Thus we can reject the null hypothesis of no orbital influence with a very high level of confidence ( $\mathrm{p}$-value $=0.00200$, or the $99.8 \%$ confidence level; Figure 6). Evaluation of the full range of sedimentation rates permitted by biostratigraphic constraints at Ceara Rise refines this estimate, indicating an optimal fit at $2.95 \mathrm{~cm} / \mathrm{ka}$ $\left(\mathrm{ASM}=1.024 \times 10^{-3}\right.$ cycles $/ \mathrm{ka} ; \mathrm{p}$-value $\left.=0.00158\right) . \mathrm{A}$ comprehensive analysis of the entire Ceara Rise $\delta^{18} \mathrm{O}$ record, spanning 18-27 Myr, is the focus of a companion study (in preparation).

\section{Conclusions}

[28] Accurate characterization of noise processes from paleoclimate data is inherently important for evaluating the physical basis of stochastic climate change [Hasselmann, 1976], and its potential links to deterministic processes. In this regard, the noise processes alone can reveal key insights into the dynamics of paleoclimate [Huybers and Curry, 2006; Meyers and Hinnov, 2010]. However, correct characterization of the noise is also requisite for discriminating between stochastic and deterministic hypotheses of climate change, such as the existence of an astronomical influence as preserved in the sedimentary archive. The null "noise" hypothesis most often utilized in cyclostratigraphic research is based upon an autoregressive-1 (AR1) red noise model, a simple stochastic process that is physically motivated by climate and depositional system dynamics, and is capable of generating cyclic variability. Previous work has demonstrated that empirically estimated AR1 model parameters ( $\rho$, the lag-1 autocorrelation coefficient) can be strongly biased when determined from data that also contain periodic/ quasiperiodic signals, a consequence of which is biased confidence levels. To address this issue, robust noise estimation techniques [Mann and Lees, 1996] have been developed, which are now commonplace in cyclostratigraphic research.

[29] In this study, ensemble model simulations indicate that the ML96 robust AR1 method is generally preferable for estimation of $\rho$, and this approach also has higher statistical power - that is, the ML96 method demonstrates a greater ability to accurately identify astronomical signals when they are in fact present in the data. However, the experiments also highlight some important limitations in the ML96 robust method with regards to cyclostratigraphic applications, including inflated confidence level estimates (greater than expected false positive rate) and excessive clumping of false positives within particular portions of the spectrum. The latter issue can impose "significant" eccentricity band signals, even in the case of pure noise, and/or may result in missing stratigraphic rhythms that truly are statistically distinguishable from AR1 noise.

[30] An alternative noise estimation method is proposed, based on a robust locally weighted regression estimate of the power spectrum (termed LOWSPEC), which overcomes these problems while achieving high statistical power. The LOWSPEC approach is flexible and can quite easily accommodate a wide range of noise backgrounds via the implementation of appropriate pre-whitening procedures. Application of the method to un-tuned (depth-scaled) $\delta^{18} \mathrm{O}$ data from Miocene sediments of the Ceara Rise indicates 
statistically significant spectral power at frequencies that are consistent with published astrochronologies [e.g., Weedon et al., 1997; Pälike et al., 2006]. Furthermore, evaluation of the frequency arrangement of these significant spatial bedding periods, using the average spectral misfit method for astrochronologic testing [Meyers and Sageman, 2007], reveals that the null hypothesis of no orbital influence can be rejected with a high degree of confidence (the $99.8 \%$ confidence level).

\section{Appendix A: ML96 Robust Red Noise Parameters and Noise Simulation Details}

[31] Application of the ML96 robust AR1 method to a given power spectrum involves selection of a median smoothing window size, and selection of an analytic fit to linear or log-transformed power [Mann and Lees, 1996]. The permissible median smoothing window size can range from $b^{*} f_{R}$ to $1 / 4^{*} f_{N}$ (where $b$ is the time-bandwidth product of the MTM analysis, $f_{R}$ is the Rayleigh frequency, and $f_{N}$ is the Nyquist frequency). Alternatively, the lower bound should be replaced by the expected bandwidth of the hypothesized climate signal (e.g., precession) if it is larger than $b^{*} f_{R}$. As implemented in the SSA-MTM Toolkit (http://www.atmos.ucla.edu/tcd/ssa/), and also in the FORTRAN code available at http://www.meteo.psu.edu/ mann/ Mann/tools/tools.html, the default values for the two ML96 robust AR1 parameters were utilized in the present study. These default values are an analytic fit to log-power, using a median smoothing window size of $1 / 5^{*} f_{N}$. Following the ML96 algorithm [Mann and Lees, 1996], the size of the median smoothing window is truncated to include fewer points near the edges of the spectrum - in this case, the lower and upper $1 / 5$ of the spectrum. The 'edge effect' introduced by this truncation approach is the key factor responsible for excess false positive rates at low frequencies when $\rho$ is large (Figures $1 \mathrm{~b}, 1 \mathrm{c}$, and $2 \mathrm{~d}$ ).

[32] The ML96 routine implemented in the SSA-Toolkit does not include the Nyquist frequency in the analytic fit, a procedure that we follow here as well. However, in contrast to the ML96 routine implemented in SSA-MTM toolkit, we also exclude the zero frequency $[\mathrm{f}(0)]$ from the fit. We choose to eliminate $f(0)$ and $f_{N}$ from the analytical fit because the theoretical distribution of power at these frequencies (more specifically, the degrees of freedom) is distinct from that of all other frequencies in the power spectrum. This is also consistent with the standard procedure of removing the mean value from the data series prior to its evaluation [Weedon, 2003].

[33] For the simulations implemented in this study, special attention has been given to the generation of random sequences that are suitable for time series spectrum estimation. Deficiencies in random number generators [Park and Miller, 1988] can result in the contribution of excess false positive detections, independent of the specific background estimation methodology. To guard against this potential limitation, we utilize the following approach: (1) two individual sets of random sequences (of same the length) are generated using the random number generator of Matsumoto and Nishimura [1998], (2) the first random sequence is sorted, and its permutation vector is used to reorder the second random number sequence.

\section{Appendix B: Details on the LOWSPEC Algorithm}

[34] The complete LOWSPEC method is comprised of four steps: (1) an initial pre-whitening, (2) MTM power spectrum estimation, (3) robust locally weighted estimation of the spectral background, and (4) assignment of confidence levels based on the estimated background. For this study, pre-whitening has been conducted using the conventional AR $1 \rho$ estimator, a lag-1 autocorrelation. This approach is selected to specifically test against an AR1 model, but other pre-whitening approaches are available [Percival and Walden, 1993; Weedon, 2003] and may be more appropriate for a given stratigraphic data set. Quantitative metrics are available to aid in determination of the most suitable pre-whitening procedure (e.g., Akaike's information criterion [Akaike, 1974]), and visual inspection of the estimated background should provide a clear indication of whether a particular pre-whitening method is appropriate.

[35] Power spectral estimation is conducted using the multitaper method of Thomson [1982]. The Thomson MTM approach is favorable because it provides an optimal tradeoff between frequency resolution, bias protection (accuracy), and consistency (precision), especially important for the evaluation of the spectral continuum (the noise background; compare Figures 203, 340 and 341 in Percival and Walden [1993]). The method obtains superior broadband bias protection and consistency via the application of multiple orthogonal tapers (discrete prolate spheroidal sequences [Slepian, 1978]) that provide an optimal concentration of power within a specified frequency bandwidth. MTM spectral estimation is implemented using the R library "multitaper." Other spectral techniques can be used for LOWSPEC, including those suitable for unevenly sampled data [Scargle, 1989], however it should be noted that the robust locally weighted background estimation procedure used here has been optimized for the MTM method. Alternative optimizations may be more appropriate for other spectral methods.

[36] Background estimation is conducted using a robust locally weighted regression procedure (equation (3)) [Cleveland, 1979] as further developed by Ruckstuhl et al. [2001] and implemented in the R library "baseline." In contrast to the original method of Cleveland [1979], this procedure utilizes asymmetric robustness weights $(w)$, which have been demonstrated to be more appropriate for background spectrum estimation in the presence of strong deterministic peaks [Ruckstuhl et al., 2001]. For the present application, the robustness criterion (' $b$ ' in equation 6 of Ruckstuhl et al. [2001]) is optimized for both the spectral bandwidth resolution (time-bandwidth product) [Thomson, 1982] and the number of data points in the analyzed data series. Finally, confidence levels based on the LOWSPEC background are determined using a chi-square distribution [Bartlett, 1978] with $2 K$ degrees of freedom, where $K$ is the number of data tapers employed in the MTM analysis [Thomson, 1982].

[37] Acknowledgments. S. Meyers would like to than Jonathan Lees (UNC-Chapel Hill) for insightful discussion of the ML96 robust AR1 method. Comments by two anonymous reviewers substantially improved this manuscript. This study was supported by U.S. National Science Foundation grant OCE-1003603 to S. Meyers. 


\section{References}

Akaike, H. (1974), A new look at the statistical model identification, IEEE Trans. Autom. Control, 19, 716-723, doi:10.1109/TAC.1974.1100705.

Bartlett, M. S. (1978), An Introduction to Stochastic Processes with Special Reference to Methods and Applications, 388 pp., Cambridge Univ. Press, Cambridge, U. K.

Berger, A., M. F. Loutre, and J. Laskar (1992), Stability of the astronomical frequencies over the Earth's history for paleoclimate studies, Science, 255, 560-566, doi:10.1126/science.255.5044.560.

Blackman, R. B., and J. W. Tukey (1958), The Measurement of Power Spectra From the Point of View of Communication Engineering, 190 pp., Dover Publ., Mineola, N. Y.

Box, G. E. P. (1953), Non-normality and tests on variances, Biometrika 40, 318-335.

Cleveland, W. S. (1979), Locally weighted regression and smoothing scatterplots, J. Am. Stat. Assoc., 74, 829-836.

Curry, W. B., et al. (1995), Proceedings of the Ocean Drilling Program, Initial Reports, vol. 154, Ocean Drill. Program, College Station, Tex.

Ghil, M., et al. (2002), Advanced spectral methods for climatic time series, Rev. Geophys., 40(1), 1003, doi:10.1029/2000RG000092.

Gilman, D. L., F. J. Fuglister, and J. M. Mitchell Jr. (1963), On the power spectrum of 'red noise', J. Atmos. Sci., 20, 182-184, doi:10.1175/15200469(1963)020<0182:OTPSON $>2.0$. CO;2.

Hasselmann, K. (1976), Stochastic climate models: Part I. Theory, Tellus, 6 , 473-485.

Hays, J. D., J. Imbrie, and N. J. Shackleton (1976), Variations in the Earth's orbit: pacemaker of the Ice Ages, Science, 194, 1121-1132, doi:10.1126/ science. 194.4270.1121.

Hinnov, L. A. (2000), New perspectives on orbitally forced stratigraphy, Annu. Rev. Earth Planet. Sci., 28, 419-475, doi:10.1146/annurev. earth.28.1.419.

Hinnov, L. A., and R. K. Goldhammer (1991), Spectral analysis of the middle Triassic Latemar Limestone, J. Sediment. Petrol., 61, 1173-1193.

Huybers, P., and W. Curry (2006), Links between annual, Milankovitch and continuum temperature variability, Nature, 441, 329-332, doi:10.1038/ nature 04745 .

Huybers, P., and C. Wunsch (2004), A depth-derived Pleistocene age model: Uncertainty estimates, sedimentation variability, and non-linear climate change, Paleoceanography, 19, PA1028, doi:10.1029/2002PA000857.

Laskar, J., F. Joutel, and F. Boudin (1993), Orbital, precessional and insolation quantities for the Earth from $-20 \mathrm{Myr}$ to $+10 \mathrm{Myr}$, Astron. Astrophys., 270, 522-533.

Laskar, J., P. Robutel, F. Joutel, M. Gastineau, A. C. M. Correia, and B. Levard (2004), A long-term numerical solution for the insolation quantities of the Earth, Astron. Astrophys., 428, 261-285, doi:10.1051/00046361:20041335.

Malinverno, A., E. Erba, and T. D. Herbert (2010), Orbital tuning as an inverse problem: Chronology of the early Aptian oceanic anoxic event 1a (Selli Level) in the Cismon APTICORE, Paleoceanography, 25, PA2203, doi:10.1029/2009PA001769.

Mann, M. E., and J. M. Lees (1996), Robust estimation of background noise and signal detection in climatic time series, Clim. Change, 33, 409-445, doi:10.1007/BF00142586.

Matsumoto, M., and T. Nishimura (1998), Mersenne Twister: A $623-$ dimensionally equidistributed uniform pseudo-random number generator ACM Trans. Model. Comput. Simul., 8, 3-30, doi:10.1145/ 272991.272995

Meyers, S. R. (2008), Resolving Milankovitchian controversies: The Triassic Latemar Limestone and Eocene Green River Formation, Geology, 36, 319-322, doi:10.1130/G24423A.1.

Meyers, S. R., and L. A. Hinnov (2010), Northern Hemisphere glaciation and the evolution of Plio-Pleistocene climate noise, Paleoceanography, 25, PA3207, doi:10.1029/2009PA001834

Meyers, S. R., and B. B. Sageman (2007), Quantification of deep-time orbital forcing by average spectral misfit, Am. J. Sci., 307, 773-792, doi:10.2475/05.2007.01.

Meyers, S. R., and L. A. Hinnov (2011), An Orbital beat in the equatorial Atlantic $(\sim 18-27 \mathrm{Ma})$ : Reliable chronometer or wishful thinking? Abstract PP13A-1805 presented at 2011 Fall meeting, AGU, San Francisco, Calif., 5-9 Dec.
Meyers, S., B. Sageman, and L. Hinnov (2001), Integrated quantitative stratigraphy of the Cenomanian-Turonian Bridge Creek Limestone Member using evolutive harmonic analysis and stratigraphic modeling, J. Sediment. Res., 71, 628-644, doi:10.1306/012401710628.

Moore, M. I., and P. J. Thomson (1991), Impact of jittered sampling on conventional spectral estimates, J. Geophys. Res., 96(C10), 18,519-18,526, doi:10.1029/91JC01623.

Mudelsee, M. (2010), Climate Times Series Analysis: Classical Statistical and Bootstrap Methods, 474 pp., Springer, Dordrecht, Netherlands.

Muller, R. A., and G. J. MacDonald (2000), Ice Ages and Astronomical Causes, 318 pp., Springer, Chitchester, U. K.

Pälike, H., J. Frazier, and J. C. Zachos (2006), Extended orbitally forced palaeoclimatic records from the equatorial Atlantic Ceara Rise, Quat. Sci. Rev., 25, 3138-3149, doi:10.1016/j.quascirev.2006.02.011.

Park, J., and T. Herbert (1987), Hunting for paleoclimatic periodicities in a geologic time series with an uncertain time scale, J. Geophys. Res., 92, 14,027-14,040, doi:10.1029/JB092iB13p14027.

Park, S. K., and K. W. Miller (1988), Random number generators: Good ones are hard to find, Commun. ACM, 31, 1192-1201, doi:10.1145/ 63039.63042

Percival, D. B., and A. T. Walden (1993), Spectral Analysis for Physical Applications: Multitaper and Conventional Univariate Techniques, 583 pp., Cambridge Univ. Press, Cambridge, U. K., doi:10.1017/ CBO9780511622762.

Ruckstuhl, A. F., M. P. Jacobson, R. W. Field, and J. A. Dodd (2001), Baseline subtraction using robust local regression estimation, J. Quant. Spectrosc. Radiat. Transf., 68, 179-193, doi:10.1016/S0022-4073(00) 00021-2.

Sadler, P. M., and D. J. Strauss (1990), Estimation of completeness of stratigraphical sections using empirical data and theoretical models, J. Geol. Soc., 147, 471-485, doi:10.1144/gsjgs.147.3.0471

Sageman, B. B., J. Rich, M. A. Arthur, G. E. Birchfield, and W. E. Dean (1997), Evidence for Milankovitch periodicities in Cenomanian-Turonian lithologic and geochemical cycles, Western Interior U.S, J. Sediment. Res, 67, 286-301.

Scargle, J. D. (1989), Studies in astronomical time series analysis. III. Fourier Transforms, autocorrelation functions, and cross-correlation functions of unevenly spaced data, Astrophys. J., 343, 874-887, doi:10.1086/ 167757.

Shackleton, N. J., S. J. Crowhurst, G. P. Weedon, and J. Laskar (1999), Astronomical calibration of Oligocene-Miocene time, Philos. Trans. $R$ Soc. London, Ser. A, 357(1757), 1907-1929, doi:10.1098/rsta.1999.0407. Slepian, D. (1978), Prolate spheroidal wave functions, Fourier analysis, and uncertainty. V: The discrete case, Bell Syst. Tech. J., 57, 1371-1429.

Thakur, T. R., and A. E. Scheidegger (1970), Chain model of river meanders, Water Resour. Res., 12, 25-47.

Thomson, D. J. (1982), Spectrum estimation and harmonic analysis, Proc. IEEE, 70, 1055-1096, doi:10.1109/PROC.1982.12433.

Thomson, D. J. (1990), Quadratic-inverse spectrum estimates: Applications to palaeoclimatology, Philos. Trans. R. Soc. London, Ser. B, 332, 539-597, doi:10.1098/rsta.1990.0130.

Thomson, D. J. (2010), Time-series analysis of paleoclimate data, in Encyclopedia of Paleoclimatology and Ancient Environments, edited by V. Gornitz, pp. 949-959, Springer, New York.

Thomson, D. J., L. J. Lanzerotti, and C. G. Maclennan (2001), The interplanetary magnetic field: Statistical properties and discrete modes, J. Geophys. Res., 106, 15,941-15,962, doi:10.1029/2000JA000113.

Vaughan, S., R. J. Bailey, and D. G. Smith (2011), Detecting cycles in stratigraphic data: Spectral analysis in the presence of red noise, Paleoceanography, 26, PA4211, doi:10.1029/2011PA002195.

Weedon, G. P. (2003), Time-Series Analysis and Cyclostratigraphy, 259 pp., Cambridge Univ. Press, Cambridge, U. K., doi:10.1017/ CBO9780511535482.

Weedon, G. P., and H. C. Jenkyns (1999), Cyclostratigraphy and the Early Jurassic timescale: Data from the Belemnite Marls, Dorset, southern England, Geol. Soc. Am. Bull., 111, 1823-1840, doi:10.1130/00167606(1999) $111<1823:$ CATEJT $>2.3$. CO;2.

Weedon, G. P., N. J. Shackleton, and P. N. Pearson (1997), The Oligocene time scale and cyclostratigraphy on the Ceara Rise, Western Equatorial Atlantic, Proc. Ocean Drill. Program Sci. Results, 154, 101-114. 